\title{
MISCELÁNEA
}

\section{NOTICIA SOBRE UN NUEVO MANUSCRITO DE LAS FIRMEZAS DE ISABELA DE GONGORA: 2 MS DP3 F3 (MS UCB 143) VOL. 165}

\author{
Antonio Cortijo Ocaña. \\ University of California, Berkeley.
}

Las presentes notas quieren dar noticia de un Ms. desconocido hasta ahora de la comedia de Góngora Las firmezas de Isabela. Ésta se halla copiada en los fols. 182-227 de un volumen misceláneo perteneciente a los fondos de la Fernán Núñez Collection en la Bancroft Library de la University of California at Berkeley (vol. 165) ${ }^{1}$. De la comedia se conocen varias ediciones y cinco manuscritos: $\mathrm{CH}$ (Ms. Chacón), E (Ms. Estrada), I (Ms. Iriarte), L (Ms. 4269 B. N. M.) y NB (Ms. 84-2-9 (445-11) de la Biblioteca Colombina).

El Ms. de la UCB contiene $111+227+111$ hojas. Mide 315 por $215 \mathrm{~mm}$. Letra del siglo xvir. Presenta señales de diferentes foliaturas. Faltan numerosos folios y un gran número de ellos está en blanco. Al menos existen dos manos: la primera, posiblemente autógrafa del maestro Blas López de Miranda, copia los folios 1-181; la segunda copia la comedia Las firmezas de Isabela del folio 182 al 227 . En el folio $1 \mathrm{v}$, margen superior izquierdo, figura el siguiente sello: "Para despachos de oficio dos m[a]r[avedi]s. Año de 1639. | Sello quarto. Año de mil seis | cientos y treinta y nueue." Las cartas de los folios 10, 12 y 15 tienen fecha de 3 de septiembre de 1639, 8 de mayo de 1638 y 9 de mayo de 1638 , respectivamente.

Los folios 1-181 corresponden a una "Pedagogía latina" del maestro Blas

1 Los fondos de la Fernán Núñez Collection sólo cuentan con unos ficheros recopilatorios parciales debidos al profesor Charles Faulhaber. En la actualidad están siendo catalogados por Ignacio Díez y Antonio Cortijo. Entre sus numerosas obras desconocidas figuran varios cancioneros de los siglos xvI y xvII (Cancionero de Fernando Carenas, Romancero espiritual, etc.) y noticias sobre diversas representaciones dramáticas del Siglo de Oro (Aventura de la roca de la competencia de Marte y Minerva). 
López de Miranda ${ }^{2}$. Existen numerosos folios en blanco y señas de comentarios latinos inacabados. Podría tratarse del "cuaderno de clase" del profesor Blas López. Una carta con encabezamiento "lectori" podria dar pie para creer que los textos y comentarios pensaban darse a la imprenta. El contenido de estos folios es el que sigue:

- [1v.] Satira 9 de Juvenal. [Comentario.]

- [3r.] Sat[ira] 9 de Juvenal. [Comentario.]

- [5v.] Del Genitivo. [Falta un folio al menos. Comentario sobre los diferentes casos latinos.]

- [7r.] Sat[ira] 1 de Juvenal. [Comentario.]

- [9v.] Sat[ira] 5. Persio. [Comentario. Abarca hasta el folio 15r. Incluye los folios 10,12 y 15 en los márgenes.]

- [10r.] [Carta de Pedro Tello a Blas López:] La semana passada reciui vna de $v$. $m$. en que me decia...

- [12r.] [Carta de Grabriel Gajor a Blas López:] S[eño]r Ma[estro] Blas Lopez. La de v. m[erce]d recibi y le certifico a v. m[erce]d tube mucho gusto en saber...

- [15r.] [Carta de Jacinto Tabernier a Blas López:] Tan confiado en su piadoso natural como en la...

- [15v.] Satura 6 de Jubenal. [Comentario.]

- [16r.] Lib[er] 6. [Versus] 430. [Comentario del libro 6 de la Eneida. Falta el inicio.]

- [18r.] Notae in lib[rum] 7 Aeneidos. [Comentario.]

- [20r.] In 8 Aeneidos notae. [Comentario.]

- [26r.] In primum librum Aeneidos | notae. [Comentario.]

- [34r.] Ad 2 Aene[i]dos notae. [Comentario.]

- [39r.] In $3 \mathrm{Ae}$ [ne]idos notae. [Comentario.]

- [42r.] In 4 Aeneidos notae. [Comentario.]

- [46v.] In lib[rum] 5 Aeneidos notae. [Comentario.]

- [48v.] In lib[rum] 6 Aen[eidos] not[ae]. [Comentario.]

- [50r.] Magistri Blasij Lopij Mirandaei | Salmanticae in Academia primarij | Latinorum librorum interpretis | calathiscus. | Novus auctorum factus interpres maligni alicuius... [Carta-prólogo a todos los comentarios del Ms., quizá reunidos con el propósito de la publicación.]

- [50v.] Ad Virgilium poetarum | principem notae. Eclog[a] 1. [Comentario.]

2 En Nicolás Antonio, Bibliotheca Hispara Nova, I (Matriti, apud Joachinum de Ibarra), se lee: "Blasius Lopez, grammaticae artis atque humanioris literaturae professor apud Salmantinos primarius, edidit Trochaicos versus ad orationis Latinae cognitionem, Salmanticae, 1634." 
- [53v.] In Eclog[am] 2 notae. [Comentario.]

- [55v.] In Eclog[am] 3 notae. [Comentario.]

- [57v.] In Eclog[am] 4 [notae.] [Comentario.]

- [58v.] In Eclog[am] 5 [notae.] [Comentario.]

- [59r.] In Eclogam 6 [notae.] [Comentario.]

- [59r.] In Eclog[am] 7 [notae.] [Comentario.]

- [60r.] In Eclog[am] 8 [notae.] [Comentario.]

- [60v.] In Eclogam 9 [notae.] [Comentario.]

- [60v.] Ecloga 10. [Comentario.]

- [61.] In 1 Georg[icon] notae. [Comentario.]

- [64v.] In Georg[icon] 2 [notae.] [Comentario.]

- [67r.] In lib[rum] 3 Georg[icon] [notae.] [Comentario.]

- [68v.] In lib[rum] 4 Geor[icon] [notae.] [Comentario.]

- [72r.] Gratulatio ad regem Academiae nomine. | Illa ego Baccaeis doctrinae mater in oris. [Dísticos elegíacos.]

- [73r.] Genethliacon carmen in principis natali. | Quale Siracosio deductum carmen ab antro. [Dísticos elegíacos.]

- [74r.] Divo Ignatio Loiolidae carmen. | Labritio quondam iuveni dum Celta superbum. [Dísticos elegíacos.]

- [75r.] Divo Francisco Xavierio | ulcera lambenti | epigramma. Accessit laturus opem Franciscus ab aedes. [Dísticos elegíacos.]

- [77r.] Exercicios I classe. $\mid$ El primer dia se les ha de dar a entender como oration es esto q[ue] ... [Se trata de una breve pedagogía escolar, seguida de una breve gramática.]

- [78v.] Las señas por donde se conocen las partes de la or[ati]on son estas. [Gramática sobre las partes de la oración.]

- [87r.] De las kalendas. | Abrijunsetino treynta | los demas a treynta y vno. [Nueve coplas sobre el modo de escribir la fechación en latín, seguidas de un comentario en prosa a las mismas con ejemplos.]

- [98r.] De metrica. Metrum carmen y versus todo es vna cosa aun$\mathrm{q}[\mathrm{ue}]$... [Pequeño tratado de métrica que cuenta con las siguientes partes: "De scansione"; "De depositione"; "De genere carminum"; "De iambico".]

- [101r.] Variae sunt carminum appel | lationes nam dicitur vel [llave] a numero versui | a numero sylla | barum | a compositione | a reuersione | a mensura | a pede crebriore | a depositione | a materia | a inventore. [Tabla-clasificación de los diferentes tipos de metros.]

- [101v.] Iambicorum metrorum species. [Catálogo de metros yámbicos con indicación de la cantidad silábica.]

- [108r.] Vers[us] 50. [Comentario a la Égloga primera de Virgilio. Falta el inicio.] 
- [108v.] Ecloga 2. [Comentario.]

- [109v.] Ecloga 3. [Comentario.]

- [111r.] Ecloga 4. [Comentario.]

- [111v.] Ecloga 5. [Comentario.]

- [112r.] Ecloga 6. [Comentario.]

- [112r.] Ecloga 7. [Comentario.]

- [112v.] Ecloga 8. [Comentario.]

- [112v.] Ecloga 9. [Comentario.]

- [113r.] Ecloga 10. [Comentario.]

- [113v.] Georgicon lib[er] 1. [Comentario.]

- [115r.] Georgicon lib[er] 2. [Sólo figura el epigrafe, no el comentario.]

- [120r.] Lib[er] 1. Od[a] 29. [Falta el inicio. Es un comentario a las

Odas de Horacio.]

- [120v.] Lib[er] 2. Od[a] 1. [Comentario.]

- [122r.] Lib[er] 3. Od[a] 1. [Comentario.]

- [124v.] Lib[er] 4. Od[a] 1. [Comentario.]

- [126r.] Liber Epodon. Antequam loca quae in hoc libro difficillima sunt aggrediar ... [Carta-declaración de los intereses exegéticos de Blas López.]

- [142r.] Magistri Blasij Lopij Mirandaei | Salmanticae in Academia primarij | auctorum Latinorum interpretis | calathiscus. | Lectori. | Maligni alicuius interpretis novus auctorum factus inter ... [Esta carta indica que el Ms. podria ser una primera redacción de lo que pensaba destinarse a la imprenta.]

- [143r.] Ad Virgilium poetarum principem $\mid$ notae. $\mid$ Eclog[a] 1. [Comentario. Siguen ahora comentarios a las Églogas, a las Geórgicas y a la Eneida, distintos en parte de los de folios precedentes.]

- [146v.] In Eclog[am] 2. [Comentario.]

- [148v.] In Eclogam 3. [Comentario.]

- [151r.] In Eclogam 4. [Comentario.]

- [152r.] In Eclogam 5. [Comentario.]

- [152v.] In Eclog[am] 6. [Comentario.]

- [152v.] In Eclog[am] 7. [Comentario.]

- [153v.] In Eclog[am] 9. [Comentario.]

- [154r.] In Eclog[am] 10. [Comentario.]

- [154v.] Notae ad libros Georgicon. I In Geor[icon] 1. [Comentario.]

- [157v.] In Georg[icon] lib[rum] 2. [Comentario.]

- [159v.] Lib [er] 3 Georgicon. [Comentario.]

- [161v.] Lib[er] 4 Georg[icon.] [Comentario.]

- [164r.] Ad Aeneidos lib[rum] 1. [Comentario.]

- [170v.] In lib[rum] 2 Aen[eidos]. [Comentario.] 
- [174r.] In 3 Aeneidos librum. [Comentario.]

- [177r.] In lib[rum] 4 Aeneidos. [Comentario.]

Los fols. 182-227 contienen la comedia Las firmezas de Isabela: "Comedia de las firmezas | de Isabela. Por d[on] Luis de | Gongora”. Cuentan con doble foliatura (la original empieza desde el número 1). La letra y la tinta son distintas de las del resto del Ms. La letra es del siglo xvir, primera mitad. La comedia está copiada a una columna. salvo en el último folio, que está a dos.

El cotejo con otras versiones y ediciones de la comedia permite extraer ciertas conclusiones ${ }^{3}$. Jammes ofrece en su edición de Las firmezas un aparato de variantes a partir de 13 fuentes, impresas y manuscritas. El Ms. de la UCB está emparentado con el qc (Qvatro comedias de diversos autores ..., Córdoba, 1613, y Madrid, 1617), que correspondian hasta ahora a una rama independiente en el stemma. Ambos, el Ms. qc y el de la UCB, deben remontarse a un antecedente común, pues presentan suficientes variantes comunes a los dos como para distinguirse de los demás y suficientes variantes entre ambos como para descartar que sean copia el uno del otro. Por otra parte, el Ms. de la UCB fue corregido con posterioridad al momento de copia: hay palabras tachadas sustituidas por otras escritas en el margen. Todas estas correcciones coinciden con variantes comunes al resto de los Mss. y ediciones, salvo en dos casos:

v. 1083: rama verde $\mathrm{CH}, \mathrm{E}, \mathrm{fd}, \mathrm{h}, \mathrm{I}, \mathrm{L}, \mathrm{mg}, \mathrm{NB}$ ramas verdes $\mathrm{UCB}$ (corregido por ramo verde) ramo verde qc.

v. 2607: A tiempo le desviara $\mathrm{CH}, \mathrm{E}, \mathrm{fd}, \mathrm{h}, \mathrm{I}, \mathrm{L}, \mathrm{mg}, \mathrm{NB}$, qc $A$ tiempo me desviara UCB (corregido por $A$ tiempo se desviara).

Dentro de las variantes significativas pueden citarse las siguientes:

v. 61 : si a tanta merced $\mathrm{CH}, \mathrm{E}, \mathrm{fd}, \mathrm{h}, \mathrm{I}, \mathrm{L}, \mathrm{mg}, \mathrm{NB}$ pues tanta merced $\mathrm{qc}$ UCB.

v. 128: "Aparte" (suprimiendo la acotación del v. 136) qc UCB.

v. 142: "Sale Tadeo" qc UCB.

v. 286: "Sale Violante $y$ dice aparte" qc UCB.

v. 297: cuerdas $\mathrm{CH}, \mathrm{E}, \mathrm{fd}, \mathrm{h}, \mathrm{I}, \mathrm{L}, \mathrm{mg}, \mathrm{NB}$ lindas qc UCB (corregido por cuerdas).

v. 534: mostrar $\mathrm{CH}, \mathrm{E}, \mathrm{fd}, \mathrm{h}, \mathrm{I}, \mathrm{L}, \mathrm{mg}, \mathrm{NB}$ mostrando qc UCB.

v. 1252: se encienda $\mathrm{CH}, \mathrm{E}, \mathrm{fd}, \mathrm{h}, \mathrm{I}, \mathrm{L}, \mathrm{mg}$, NB se emprenda qc UCB.

3 El cotejo se puede realizar a partir de la edición de Robert Jammes, Las firmezas de Isabela, Madrid, Castalia, 1984, cuyo aparato crítico ocupa las páginas 286-305. 
RFE, LXXV, 1995

v. 2603: la berengena $\mathrm{CH}, \mathrm{E}, \mathrm{fd}, \mathrm{h}, \mathrm{I}, \mathrm{L}, \mathrm{mg}, \mathrm{NB}$, qc las berengenas $\mathrm{UCB}$.

v. 157: con otro en secreto $\mathrm{CH}, \mathrm{E}, \mathrm{fd}, \mathrm{h}, \mathrm{I}, \mathrm{L}, \mathrm{mg}, \mathrm{NB}$ con alguien en secreto qc con alguien secreto $\mathrm{UCB}$.

vv. 162-5: (Faltan en qc, pero no en UCB).

v. 197: por otro modo $\mathrm{CH}, \mathrm{E}, \mathrm{fd}, \mathrm{h}, \mathrm{I}, \mathrm{L}, \mathrm{mg}, \mathrm{NB}, \mathrm{UCB}$ por otro rumbo qc.

v. 1955: (En qc la réplica de Isabela se prolonga hasta 1956 y es interrogativa). 